\title{
Téoros
}

Revue de recherche en tourisme

\section{Les stratégies de marketing de Tourisme Québec sur le marché américain}

\section{Barbara Di Stéphano}

Volume 9, numéro 2, juillet 1990

Le marché américain

URI : https://id.erudit.org/iderudit/1080029ar

DOI : https://doi.org/10.7202/1080029ar

Aller au sommaire du numéro

Éditeur(s)

Université du Québec à Montréal

ISSN

0712-8657 (imprimé)

1923-2705 (numérique)

Découvrir la revue

Citer cet article

Di Stéphano, B. (1990). Les stratégies de marketing de Tourisme Québec sur le marché américain. Téoros, 9(2), 23-26. https://doi.org/10.7202/1080029ar d'utilisation que vous pouvez consulter en ligne.

https://apropos.erudit.org/fr/usagers/politique-dutilisation/ 


\section{Les stratégies de marketing de Tourisme Québec sur le marché américain}

Le marché américain correspond sans nul doute à l'un des marchés touristiques les plus prometteurs tant en termes de volumes que de recettes. On estime ces dernières à plus de 30 milliards de dollars américains en 1999. Toutefois, la part laissée aux voyages internationaux estnettement moins impressionnante puisque malgré un taux de départ de $66 \%$, les Américains réalisent plus de $96 \%$ de leurs déplacements touristiques dans leur propre pays.

Bien que les prévisions pour $1^{\prime}$ an $2000^{(1)}$ laissent présager des diminutions de $14 \%$ des nuitées internationales et de plus de $8 \%$ pour les recettes touristiques, le Canada, en tant que voisin immédiat, bénéficie pourtant des flux touristiques américains dans une proportionnonnégligeable: presqu'un tiers des voyages intemationaux des Américains a lieu dans une des provinces ou territoires canadiens.

Globalement, le Canada connaît, depuis ces demières années, une croissance du nombre de ses voyageurs américains sejoumant une nuit ou plus ainsi que de leurs dépenses. Malgré cela, la progression de sa part de marché est lente au profit des destinations concurrentes notamment européennes et asiatiques.

Il est intéressant de souligner qu'entre 1984 et 1988 , le Québec montre une meilleure performance que celle du Canada, affichant des résultats croissants sur ce marché et supérieurs à ceux de l'Ontario et de I'Ouest du pays.

Les flux touristiques américains, d'est vers l'est et d'ouest vers l'ouest, expliquent cette situation favorable. Les régions les plus émettrices de touristes intemationaux demeurent la Nouvelle-Angleterre, l'Atlantique Centre, le Centre Nord-Est et le Pacifique. De ces quatre régions, trois sont situées à l'est du pays, soit à proximité de la Belle Province. De plus, le nombre de

Barbara Di Stéphano est enalyste de la Direction du Marketing du miniatère du Touriame du Cuébee. voyages d'Américains du Nord-Est a progressé plus vite que celui des autres régions, et ce, pour des destinations limitrophes comme la province de Québec.

Considérant ce bassin important de voyageurs potentiels et la croissance de leurs voyages, il est aisé de comprendre ce qui justifie les choix de marchés américains cibles du ministère du Tourisme du Québec. Le gouvernement a toujours déployé une bonne partie de ses activités de marketing touristique surcinq principales régions américaines: la Nouvelle-Angleterre, le Centre Atlantique, l'Atlantique Sud, le Centre Nord-Est et l'Ouest. Au fil des ans, les stratégies ont évolué en fonction notamment des opportunités de marché, des forces concurrentielles et des disponibilités budgétaires.

Jusqu'à récemment, des bureaux touristiques étaient ouverts dans certaines régions américaines permettant une représentation de la province en tant que destination touristique. Ainsi, la présence de conseillers touristiques dans les bureaux de New York, Boston, Chicago, Atlanta et Washington favorisait les démarches commerciales telles que les participations à des foires, bourses et autres manifestations et la sollicitation auprès du réseau de distribution afin de mieux faire connaitre la destination et d'accroître le nombre de forfaits programmant le Québec.

\section{Les stratégies des années " 80}

Les stratégies de marketing développées dans les années 1980 conduisaient à positionner le Québec comme destination touristique intéressante toute l'année durant et mettait en valeur certains produits (agrément-été, plein air, ski, circuit, etc.) et les régions québécoises. En fonction des segments de clientèle visés (voyages familiaux en automobile, sportifs, skieurs, etc.), les principaux produits génériques offerts correspondaient à des activités reliées à I'histoire, à la culture, à l'architecture; à la vie urbaine, aux sports et aux événements; à la nature et à la villégiature; à la pêche, à la chasse, au ski et à la motoneige. La spécificité culturelle, l'hébergement, la gastronomie et l'accueil constituaient le cadre général dans lequel s'inscrivaient tous ces produits.

Mais les produits commercialisés correspondaient surtout à des séjours de ville, des circuits et des séjours de plein air dans différentes régions québécoises; les villes de Montréalet de Québec demeuraient les principaux points d'attrait les plus utilisés pour des expériences touristiques au Québec.

En bref, les vacances au Québec, même pour les Américains, se vendaient beaucoup plus par les régions que sous l'angle de produits touristiques. Or, parler du Québec à un Américain passe encore (avec une carte) mais il faut reconnaitre la difficulté de vendre des régions aux noms inconnus.

\section{Les campagnes antérieures}

En accord avec les perceptions des consommateurs américains vis-à-vis la destination québécoise, la différence culturelle et historique a toujours été l'élément distinctif, et c'est sur cet avantage unique que le positionnement du Québec fut amor$c e ́$ et maintenu pendant des années.

En 1985, un repositionnement de la destination, afin d'incorporer un élément d'hospitalité et de partage dans l'invitation que le Québec adressait aux Américains, permitde contrecarrerlaperceptionnégative qu'ils affichaient sur l'accueil québécois. Au chapitre de la publicité hors Québec, commença alors la période des campagnes sous la thématique "Come Say Bonjour". L'importante facette de ce repositionnement reposait sur la continuité assurée en milieu québécois par la campagne intra-Québec communiquant le message "Bonjour".

À partir de l'été 1985 donc, le Québec lance cette invitation à ses voisins de la Nouvelle-Angleterre et des régions de la côte Est. Les annonces représentent principalement le Vieux-Québec, le VieuxMontréal et les rapides de Lachine. La radio ainsi que différents quotidiens et 
magazines constituent les supports médiatiques.

Quant à lasaison hivernale, les années 1980 sont marquées par la campagne de ski "Skiing a la française". Initi6e en 1982 , cette thematique permit de percer les marchés cibles visés et conduisit à une forte notoriété de la destination pour ce produit. Des efforts furent ensuite déployés pour integrer les deux campagnes, "Come Say Bonjour" et "Skiing àla française" et assurer une transition publicitaire capitalisant sur l'effet cumulatif du message hivernal véhiculé depuis quatre ans.

Outre ces principales campagnes publicitaires se poursuivant d'une année à l'autre avec les modifications nécessaires, d'autres, de façon plus ponctuelle, ont présenté sur le marché américain les produits motoneige et congres. Le tableau synthèse nous donne un aperçu des campagnes développées depuis 1982 .

En ce qui concerne les publications, les brochures d'éveil a la destination ont toujours été d'une excellente qualité visuelle mais aux dépens d'un contenu informationnel. Elles n'ont jamais, en fait, vraiment répondu aux attentes des clientèles qui désiraient des renseignements sur un produit en particulier. Néanmoins, les guides touristiques régionaux canalisaient l'ensemble de l'information touristique.

\section{Les orientations 1989-1992 et les stratégies de mise en marché}

Le diagnostic du tourisme quebecois, pose en 1988 , démontre certaines lacunes au niveau de la mise en marché de la destination: malgré des progrès incontestables, elle demeure erratique, hesitante et insuffisamment axee sur les produits. De plus, le positionnement de la destination, malgré les campagnes publicitaires régulières, souffre d'inconstance et de confusion.

Les conclusions découlant de ce diagnostic servent alors d'assises a 1'élaboration d'un plan stratégique de marketing pour les années 1989-1992. La nouvelle stratégie propose des orientations visant a permettre à l'industrie touristique québécoise de remédier aux causes de sa faible performance et, de ce fait, d'accroitre sa part de marche.

Les régions américaines cibles sont désormais identifiés comme suit:
- marche primaire: Nouvelle-Angleterre et Centre Atlantique;

- marché secondaire: Centre Nord-Est et Atlantique Sud;

- marché tertiaire: Californie.

Arrime au processus d'achat du consommateur, le tourisme québécois privilégie dès lors une approche-produit et les efforts consentis a la promotion touristique portent sur huit produits jugés prioritaires, lesquels sont par ordre d'importance: les grandes villes, les circuits, les sejours de villégiature, les congrès et les réunions, le ski alpin, la chasseet la péche, la motoneige et $1^{y}$ aventure. $A$ ces huit produits, s'ajoute le segment des voyages-motivation.

Le positionnement choisi pour le marché s'inspire encore du caractère distinctif du Québec, tant sur le plan culturel que patrimonial.

Au niveau de la représentation sur le marche americain, les conseillers touristiques des bureaux de New York, Bostonet Chicago continuent leurs démarches commerciales.

Selon le marche, certaines activites sont privilégiées. Les marchés primaires sont abordés principalement par le biais de la publicite, des publications destinées aux consommateurs ainsi que par la demarche associative avec les partenaires de l'industrie. Les marchés secondaires, pour leurpart, sont travailles surtout de l'intérieur en associationavecles partenaires du milieu, et de l'extérieur parla promotion auprès de 1 'industrie et par les relations de presse. L'approche des marchés tertiaires met 1 'accent sur les relations de presse et sur les actions conjointes avec les collaborateurs gouvemementaux et nationaux.

En bref, plus le marché est éloigné, plus 1'usage des relations de presse et de 1 'association ainsi que l'exploitation du réseau de distribution prennent de l'importance.

Pour des fins de promotion, les produits touristiques précités sont regroupés autour des trois thèmes génériques suivants: agrément-été (grandes villes, circuits, séjours de villégiature, chasse et pêche, aventure); agrément-hiver (grandes villes, ski alpin, sejours de villégiature, motoneige, aventure); affaires (congrès et réunions, voyages-motivation). La stratégie de mise en marché met l'accent sur un bon rapport qualite-prix pour un marché de moyen a haut de gamme.
Lapublicités'oriente égalementen fonction de $1^{\prime}$ approche produit et deux campagnes "agrément-été "t "agrément-hiver" sont retenues. En parallèle à cet effort promotionnel destiné aux consommateurs, des placements publicitaires ponctuels a l'intention du réseau de distribution sont effectués dans les magazines spécialisés destinés a 1 'industrie. La publicité reste le moyen par excellence pour $s^{\dagger}$ adresser à un grand nombre de consommateurs et susciter une attitude favorable de leur part.

En vue de mieux répondre aux exigences des consommateurs, les publications institutionnelles quant à leur contenu et à leur présentation ont été modifiées. Pour la seconde anné, le ministère du Tourisme publieet distribue les brochures Destination Québec, vacances d'été et Destination Québec, vacances d'hiver, lesquelles présentent respectivement les produits de I'agrément-été et de l'agrément-hiver. Par ailleurs, une brochure de forfaits "chasse et péche" présente les forfaits disponibles pour la chasse et la pêche dans plus de 80 pourvoiries québécoises.

Des relations de presse, a 1'intention des journalistes, sont toujours de mise afin d'augmenter le nombre et la qualité des reportages touristiques sur le Québec. A cette fin, des toumes de familiarisation sont offertes aux joumalistes leurpresentant la destination et la gamme de ses produits touristiques.

Pour compléter ces différentes démarches auprès des consommateurs, une presence accrue se poursuit dans les salons specialisés offrant le plus de potentiel pour le Québec. Les activités auprès du réseau de distribution prennent la forme de séminaires, de soiresréceptions, d'ateliers et de tournees de familiarisation. Par exemple, la nouvelle bourse commerciale Bienvenue Québec, lancée en 1989-1990, met en présence les vendeurs et le réseau de distribution québécois avec les grossistes réceptifs américains. A cela s'ajoute la diffusion du bulletin Québec Grandeur Naturedestiné a l'industrie, qui renseigne sur $1^{\prime}$ offre touristique du Quebec et met l'accent tant sur les nouveaux produits que sur ceux en développement.

Les efforts consentissurle marché américain représentent plus d'un tiers du budget global de promotion, soit près de 4,5 millions de dollars. Laplus grande part est évidemment affectee aux marches primaires soit la Nouvelle-Angleterre et le Centre Atlantique, et les produits les plus mis de 1 'avant concernent les grandes villes $(\$ 1,4 \mathrm{M})$ et le 
TABLEAU 1

Synthèse - Campagnes publicitaires (marché américain) 1982-1990

\begin{tabular}{|c|c|c|}
\hline Saison & Type de campagne & Thème/Signature publicitaire \\
\hline \multirow{3}{*}{$\begin{array}{l}\text { Eté } 82 \\
\text { Hiver } \\
82 / 83\end{array}$} & Agrément-été & Québec, the vacation with la différence \\
\hline & Ski & Québec, Skiing à la française \\
\hline & Congrès & $\begin{array}{l}\text { Quebec, make yours the brightest } \\
\text { convention under the stars }\end{array}$ \\
\hline \multirow{4}{*}{$\begin{array}{l}\text { Eté } 83 \\
\text { Hiver } \\
83 / 84\end{array}$} & Positionnement & Quebec, the vacation with la differrence \\
\hline & Ski & Quebec, Skiing à la française \\
\hline & Pêche & Quebec, come share the challenge \\
\hline & Congres & $\begin{array}{l}\text { Quebec, you can't sell conventions } \\
\text { any brighter }\end{array}$ \\
\hline $\begin{array}{l}\text { Eté } 84 \\
\text { Hiver }\end{array}$ & Agrément-été & Québec, the vacation with la différence \\
\hline $84 / 85$ & Ski & Quebec, Skiing a la française \\
\hline
\end{tabular}

Hiver

$85 / 86$

Motoneige

Courts sejours

Agrément-été

Ski

Motoneige

Congrès

Eté 86

Hiver

$86 / 87$

Été 87

Hiver

$87 / 88$

Été 88

Hiver

$88 / 89$

Eté 89

Hiver

$89 / 90$

Ski

Congrès

Agrément-été

Ski

Agrément-étét

Ski

Agrément-été
Québec, the ultimate snowmobiling experience

Montréal winter break

Quebec, Come Say Bonjour

Quebec, Sking à la française

Quebec, the ultimate snowmobiling experience Come Say Bonjour

Courts sejours d'hiver Come Say Bonjour

If you don't have a clue where to meet next, here's a couple of dozen/

Come Say Bonjour

Agrément-été Come Say Bonjour

Agrement-hiver
Québec, Skiing à la française

If you dont" have a clue where to meet next, here's a couple of dozen

Québec, Come Say Bonjour

Québec, It's more than skiing

Come Say Bonjour

Quebec, It's more than skiling

Quebec, the old New World right next door/Bonjour Québec

Bonjour Québec
Support

Marché

quotidien

radio, quotidien et magazines spécialisés

magazines spécialisês

quotidiens

radio, quotidiens et magazines spécialisés

magazines spécialisés

magazines spécialisés

télévision, quotidiens

radio, quotidiens et magazines spécialisés

magazines spécialisés

quotidiens

radio, quotidiens et magazines

radio, magazines specialises, magazines d'intérêt général

magazines spécialises

quotidiens et magazines d'interet genéral magazines apécialisés

quotidiens et magarines specialises

quotidiens et magazines spécialisés télévision, quotidiens

magazines specialisés et d'intérêt général
Centre-Atlantique, Atlantique Sud, Centre Nord-Est

Centre Atlantique, Atlantique Sud, Centre Nord-Est.

Centre Sud-Ouest, (Dallas, Houston, New-Orleans)

Nouvelle-Angleterre, Centre Atlantique, Centre Nord-Est

Centre Atlantique, Atlantique Sud, Centre Nord-Est, Centre Nord-Ouest (Dallas)

Nouvelle-Angleterre, Centre Atlantique, Centre Nord-Est

Centre Atlantique, Nouvelle-Angleterre Nouvelle-Angleterre

Centre Atlantique, Atlantique Sud, Centre Sud-Ouest (Nouvelle-Orléans)

Nouvelle-Angleterre, Centre Atlantique, Centre Nord-Est Nouvelle-Angleterre, Centre-Atlantique

Centre Atlantique, Nouvelle-Angleterre

Centre-Atlantique, Nouvelle Angleterre 
ski alpin ( $\$ 1,2 \mathrm{M}$ ). II faut noter que les efforts dévolus au produit congrès sont à peu près equivalents sur les marchés primaires et secondaires alors que ceux concernant les voyages-motivation sont nettement supérieurs pour le Centre NordEst. Plus de $85 \%$ des efforts déployés sur la Californie visent spécifiquement le produit circuit.

\section{La campagne agrément-été hors Québec 1990}

Le ministère du Tourisme poursuit, pour l'été 1990, la campagne de l'année demière, qui a montré des résultats très positifs.

Les objectifs de communication consistent a:

- inciter la clientèle cible à choisir le Québec comme destination de vacances pour l'été 1990;

- augmenter la notoriété de l'ensemble du Québec auprès d'un large auditoire de touristes potentiels;

- véhiculer le positionnement de la destination à travers toute communication.

Le positionnement arrêté pour cette année est le suivant: LeQuébec, c'estl' expérience d'une culture differente, a proximité. Les phrases clés supportant le positionnement reflètent cette proximité:

- Le Québec, c'est un coin d'Europe d votre porté;

- Le Québec est a moins d" une journée de New York, Boston, ...;

- Le Québec offre un véritable dépaysement, gräce à son caractère distinctif sur les plans culturel et patrimonial.

Les adultes de 28 à 45 ans, de scolarité moyenne à élevée, bénéficiant de revenus moyens à élevés et résidant dans des agglomérations urbaines, localisées dans un rayon de 800 kilomètres autour de Montréal, représentent un des groupes cibles visés par la campagne. Les retraités et préretraités (50 ans et plus) composent le groupe cible secondaire.

Le processus décisionnel pourdes vacances se faisantenviron un à deux mois à l'avance, la publicité paraît pendant le mois d'avril 1990 dans les villes de Buffalo, New York, Rochester, Syracuse, Hartford/New Haven, Providence, Boston, Albany/Schenectady, Troy, Brimghamton, Utica, Wilkes-Barre/ Scranton, Philadelphie, Harrisburg/ Lancaster et Portland.
La télévision demeure le médium principal de la campagne et les joumaux (section voyages) sont utilisés comme support tactique à la campagne télévisée.

La télévision doit attirer l"attention du consommateur par la beauté des images mais doit surtout, dans un court laps de temps de 30 secondes, communiquer une image claire qui différencie le Québec des autres destinations nord-américaines. Le message que le consommateur retiendra est le suivant: Le Quebec, c'est un coin d'Europe a votre portée.

Les annonces publiées dans les joumaux (noir et blanc) insistent sur le caractère distinctif du Québec et sur sa proximité géographique. De plus, les annonces véhiculent le numéro 800 du Ministère afin d'inciter les demandes d'informations.

Les nouvelles orientations stratégiques de marketing prévoient également une concertation du ministère du Tourisme avec ses différents partenaires de l'industrie.

Cette concertation s'effectue principalement au niveau des produits et permet de fixer, de concert avec les intervenants du milieu, des objectifs communs et de définir les moyens à développer pour atteindre les bonnes cibles.

Ces nouveaux moyens mis en oeuvre ainsi que l'approche produit devraient permettre au tourisme québécois de retrouver un niveaud'activités acceptable etainsi réduire le deficit touristique croissant $^{(2)}$ depuis quelques années; l'objetif étant toujours d'assurer une progression des recettes touristiques. Donner une image forte et continue du Québec reste le grand souhait du Ministère. Un positionnement efficace, une offre structurée et une concertation entre les partenaires de l'industrie permettront au Québec de mieux lutter contre la concurrenceet d'accroître ses parts de marche. $f$

NOTE

(1) EDWARDS, Anthory, International Touriem Fore cavte to 1909 .

(2) En dollara coumanta. 